\title{
Au/GaAs photovoltaic structures with single-wall carbon nanotubes on the microrelief interface
}

\author{
N.L. Dmitruk ${ }^{1}$, O.Yu. Borkovskaya ${ }^{1}$, S.V. Mamykin ${ }^{1}$, T.S. Havrylenko ${ }^{1}$, I.B. Mamontova ${ }^{1}$, \\ N.V. Kotova ${ }^{1}$, E.V. Basiuk ${ }^{2}$ \\ ${ }^{I}$ V. Lashkaryov Institute of Semiconductor Physics, National Academy of Sciences of Ukraine, \\ 41, prospect Nauky, 03028 Kyiv, Ukraine; \\ Phone: +38(044)-525-64-86; e-mail: mamykin@isp.kiev.ua \\ ${ }^{2}$ Centro de Ciencias Aplicadas y Desarrollo Tecnológico, Universidad Nacional Autónoma de México (UNAM), \\ Circuito exterior C.U. 04510 México D.F., Mexico
}

\begin{abstract}
The effect of single-wall carbon nanotubes nanolayer on photoelectric properties of $\mathrm{Au} / n$-GaAs photovoltaic structure with a microrelief interface has been investigated. Microrelief interfaces of dendrite-like and quasi-grating type aimed at enhancement of photocurrent have been prepared by the wet chemical anisotropic etching of GaAs. Carbon nanotubes obtained using the arc-discharge method were deposited on GaAs surface modified with poly(vinylpyridine) by dip-coating repeated several times. Optical, photoelectric and electrical properties of Au/GaAs structures have been studied in dependence on the averaged thickness of nanotubes nanolayer. Considerable photocurrent enhancement has been determined for structures with both the flat and textured interface but with a different optimal thickness of nanotubes layer. The effect was concluded to be caused by increasing the lateral photocurrent component due to enlargement of the current collection area and increase of the light trapping.
\end{abstract}

Keywords: single-wall carbon nanotubes, poly(vinylpyridine), dimethylformamide, microrelief interface, $\mathrm{Au} / \mathrm{GaAs}$ photovoltaic structures, optoelectronics.

Manuscript received 15.08.14; revised version received 18.11.14; accepted for publication 19.02.15; published online 26.02.15.

\section{Introduction}

Carbon-based nanomaterials, fullerenes and nanotubes, have attracted great research interest for the use in optoelectronics and photovoltaics due to their unique electronic properties $[1,2]$. Enhancement of the shortcircuit photocurrent and photoconversion efficiency was found for the metal $/ n$-Si barrier structures with $\mathrm{C}_{60}$ fullerene intermediate layer [3]. Single-wall carbon nanotubes (SWCNTs) have been proposed for enhancement of the efficiency of organic solar cells [4] as well as for the inorganic ones, specifically for $\mathrm{Au} / \mathrm{Si}$ photovoltaic structures [5]. In these structures, CNTs act as transparent electrodes replacing $\mathrm{In}_{2} \mathrm{O}_{3}: \mathrm{Sn}$ (ITO) or semitransparent metal layers, and promoting an increase of the collection area for current carriers photogenerated in semiconductor. At the same time, the optical and conduction properties of the network layers made of SWCNTs were shown to depend both on technology of CNT layer formation and deposition on the substrate as well as on the structure and adhesion properties of substrate [1, 2, 5-8]. So, the elaborated by us method of SWCNT nanolayer deposition on the $\mathrm{Si}$ surface modified with poly(vinylpyridine) (PVP) for $\mathrm{Au} / n-\mathrm{Si}$ photovoltaic structures [5] should be verified in the case of other structures. This work is aimed at the study of 
optical and photoelectric properties of Schottky barrier type $\mathrm{Au} / n$-GaAs heterostructures with SWCNTs on the flat and microrelief interface to determine the possibility of additional enhancement of photocurrent for structures with microtextured interfaces.

\section{Experimental details}

$n$-type (100) GaAs wafers with the doping impurity concentration close to $10^{16} \mathrm{~cm}^{-3}$ were used. GaAs surface microreliefs of quasi-grating and dendrite types were prepared using the wet chemical anisotropic etching [9]. Highly purified SWCNTs were produced using the arc-discharge method. Well-sonicated in an ultrasonic bath SWCNT-dimethylformamide (DMF) mixture was used to deposit SWCNTs onto GaAs and satellite glass substrates modified with poly(vinylpyridine) (PVP)-ethanol solution in accord with the method [10]. SWCNTs were deposited using dip-coating and drying in air repeated several times. The photovoltaic structures were fabricated by evaporation of semitransparent $\mathrm{Au}$ layer (with the thickness $30 \mathrm{~nm}$ ) through the opaque mask with various diameters of openings ( 0.8 and $1.3 \mathrm{~mm})$ on GaAs surfaces with and without SWNCTs. The In ohmic contacts were fabricated on the back side of GaAs wafers.

Optical, photoelectric and electrical properties of $\mathrm{Au} / n$-GaAs structures have been studied. The shortcircuit photocurrent was measured within the $0.4 \ldots 0.9 \mu \mathrm{m}$ spectral range. Light (under simulated AMO illumination) and dark current-voltage characteristics were measured.

\section{Results and discussion}

Fig. 1 demonstrates the obtained with scanning electron microscopy (SEM) images of SWCNTs deposited on the flat semiconductor surface modified with PVP. It is seen that SWCNTs, or their bundles, have the length $\geq 1 \mu \mathrm{m}$ (Fig. 1a) and form the network on the surface of PVP layer (Fig. 1b). It should be noted that surface morphology of PVP layer has a granular or globular form, which affects SWCNT network formation. The optical microscopy images of SWCNT/PVP/GaAs structures with the flat and textured interfaces are shown in Fig. 2. It is seen that SWCNT layers are not uniform on both the flat and textured PVP/GaAs surfaces. The SWCNT network forms separate spots of increased density having different shapes and sizes. Both the number of spots and their sizes increase with increasing the dip-coating times. It is accompanied by a decrease of the light transmittance into semiconductor both through the semitransparent $\mathrm{Au}$ electrode and through the neighboring $\mathrm{PVP} / \mathrm{GaAs}$ interface. At the same time, these conducting spots forming the ohmic contact with $\mathrm{Au}$ electrode increase the area of lateral photocurrent collection. So, for total photocurrent enhancement the regime of the SWCNT deposition on semiconductor surface should be optimized.

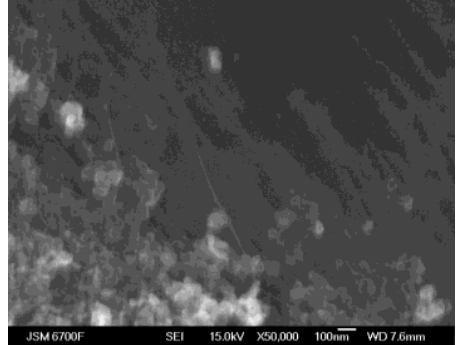

a)

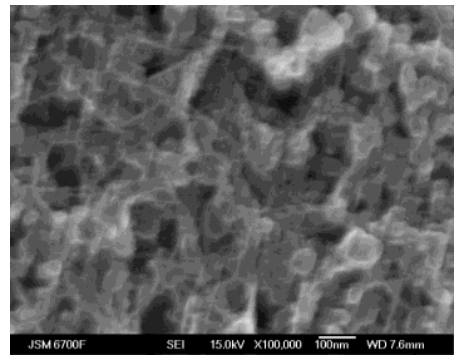

b)

Fig. 1. Scanning electron microscopy images of SWCNTs deposited on the flat semiconductor surface modified with PVP.
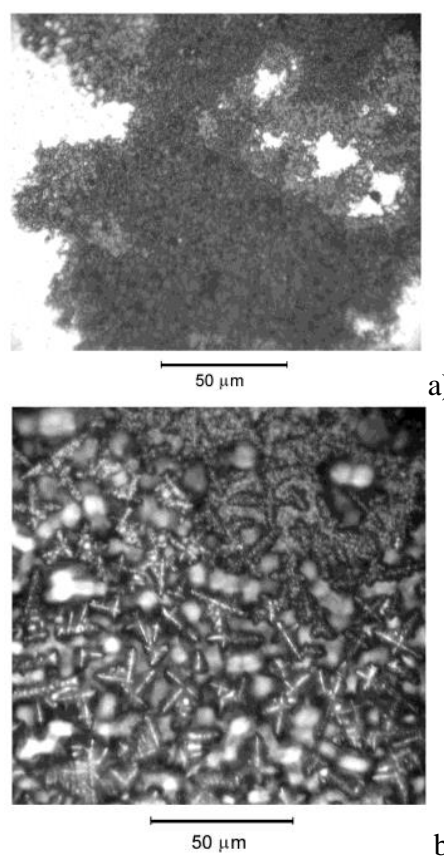

a)

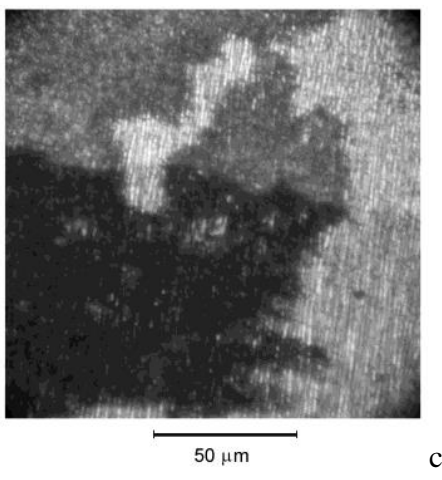

b)

c)

Fig. 2. Optical microscopy images of $\mathrm{SWCNT} / \mathrm{PVP} / \mathrm{GaAs}$ structures with the flat $(a)$ and textured interfaces of dendritelike $(b)$ and quasi-grating type $(c)$. 

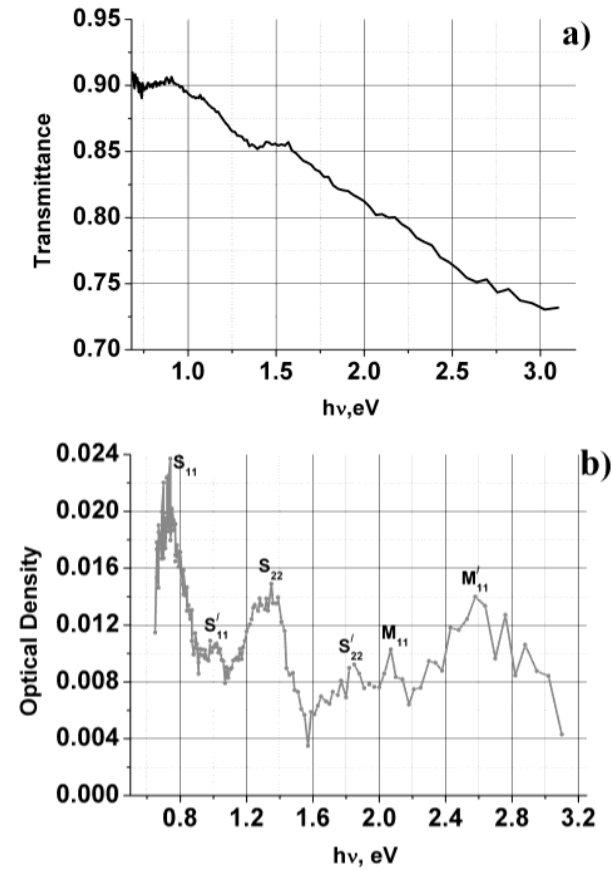

b)

Fig. 3. a) Light transmittance spectrum of SWCNT film deposited using several times repeated dip-coating on the PVPmodified glass substrate. b) Optical density spectrum for the same film obtained from optical absorption spectrum after subtraction of background absorption.

The light transmittance spectrum averaged over the SWCNT layer deposited on the glass substrate modified with PVP is shown in Fig. 3a. The analysis of the corresponding light absorption (optical density) spectrum after subtraction of the background absorption (Fig. 3b) permits one to evaluate the distribution of SWCNT diameters by comparing the obtained energy values of absorption peaks with those from the Kataura plots [6]. Two series of absorption peaks can be identified as corresponding to electronic transition between mirror-image spikes in the density of states in semiconducting tubes (for the first and second energy gaps) and metallic ones with almost the same diameters: $\mathrm{S}_{11}, \mathrm{~S}_{22}, \mathrm{M}_{11}$ and $\mathrm{S}_{11}^{\prime}, \mathrm{S}_{22}^{\prime}, \mathrm{M}^{\prime}{ }_{11}$. Estimated SWCNT diameters are grouped around 0.84 and $1.1 \mathrm{~nm}$ for both semiconducting and metallic tubes.

The spectra of the short-circuit photocurrent expressed as the external quantum efficiency normalized to the area of diode $\left(1.33 \mathrm{~mm}^{2}\right)$ are shown in Fig. 4 for $\mathrm{Au} / \mathrm{GaAs} \quad(1), \quad \mathrm{Au} / \mathrm{SWCNT} / \mathrm{PVP} / \mathrm{GaAs} \quad$ (2) and $\mathrm{Au} / \mathrm{PVP} / \mathrm{GaAs}(3)$ structures with flat $(a)$, dendrite-like $(b)$ and quasi-grating $(c)$ types of the surface microrelief. The photocurrent values for diodes of this area are mainly caused by the current carriers generated in GaAs under the semitransparent $\mathrm{Au}$ electrode. It is seen that PVP layer decreases essentially the photocurrent value, acting as isolating interlayer and weakening also the effect of SWCNTs. However, deposition of SWCNTs still increases the photocurrent, mainly in the long-wave region both for structures with flat interfaces and for microtextured ones. This effect is mainly caused by increasing the lateral component of photocurrent due to enlargement of the collection area of current carriers generated outside the edges of Au electrode. So, it must be greater for diode structures of smaller area that is seen in Fig. 5 (with the diode area close to $0.5 \mathrm{~mm}^{2}$ ). This figure demonstrates also the dependence of the photocurrent value and spectral response on the averaged thickness of SWCNT layer changed by the number of dipping times. It is seen that these dependences are different for structures with flat and textured interfaces. Specifically, a greater effect of photocurrent enhancement was obtained for structures with flat interfaces at 8-fold repeating SWCNT layer deposition but for the structures with interface microrelief of quasi-grating type at 16 -fold repetition. The same peculiarities of the SWCNT layer effect are observed for the light current-voltage characteristics presented in Fig. 6. So, the optimal thickness of SWCNT layer for maximal enhancement of the photocurrent should be chosen for the investigated structures with taking into account the character of their interface microrelief.
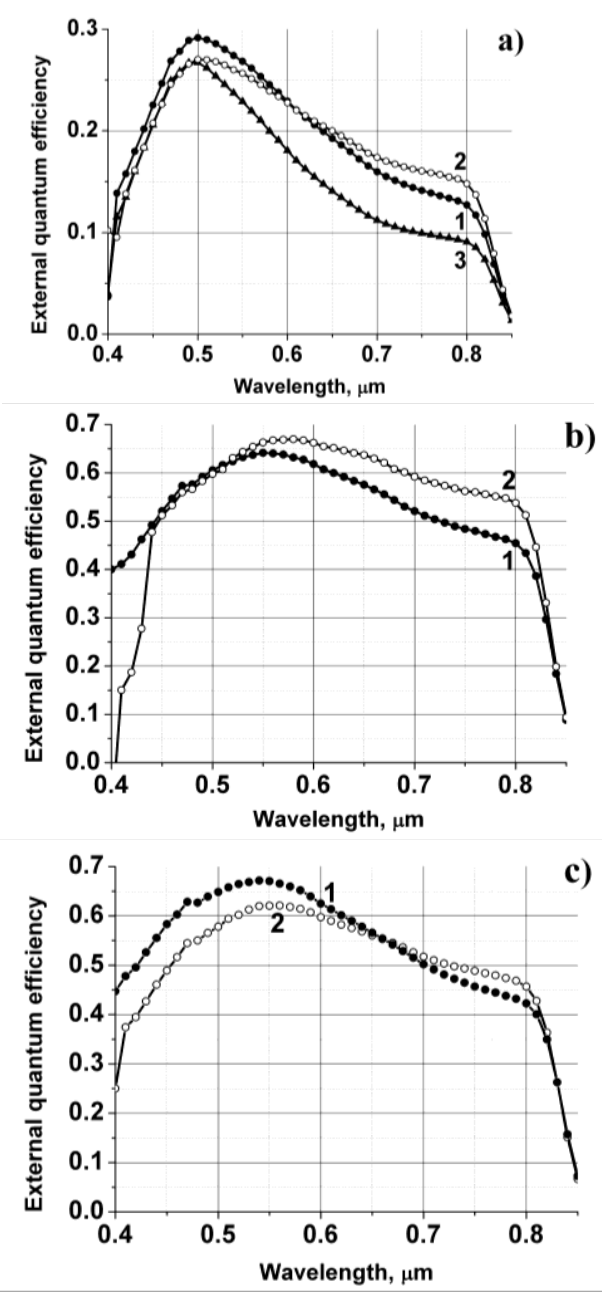

Fig. 4. Spectra of the short-circuit photocurrent expressed as the external quantum efficiency normalized to the area of diode $\left(1.3 \mathrm{~mm}^{2}\right)$ for $\mathrm{Au} / \mathrm{GaAs}$ (1), Au/CNT/PVP/GaAs (2), $\mathrm{Au} / \mathrm{PVP} / \mathrm{GaAs}(3)$ structures with flat $(a)$, dendrite-like $(b)$ and quasi-grating $(c)$ types of surface microrelief, 8-fold dip-coated with SWCNT. 

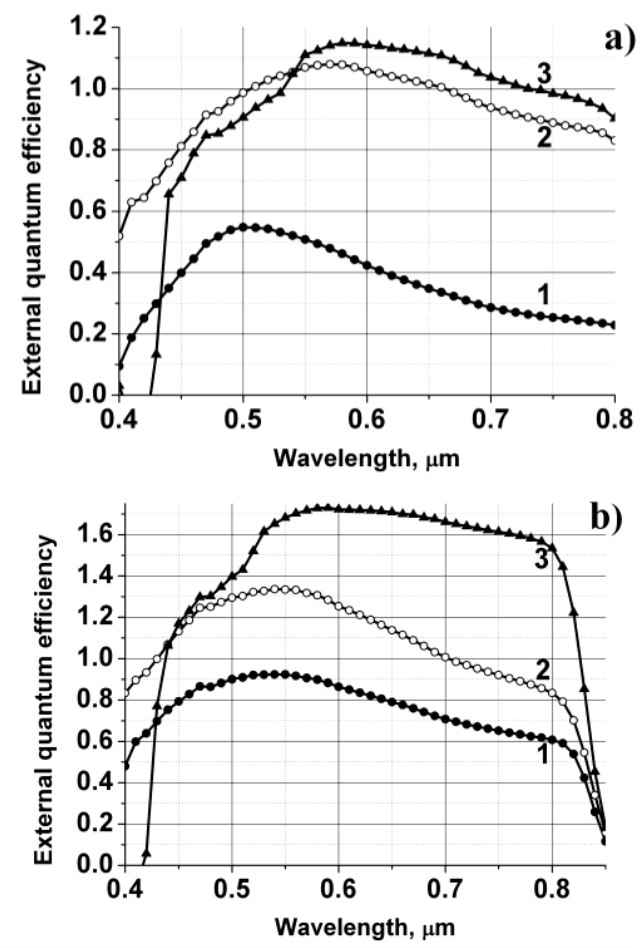

Fig. 5. The short-circuit photocurrent expressed as the external quantum efficiency normalized to the area of diode $\left(0.5 \mathrm{~mm}^{2}\right)$ for $\mathrm{Au} / \mathrm{GaAs}(1), \mathrm{Au} / \mathrm{SWCNT/PVP/GaAs}(2,3)$ structures with flat $(a)$ and quasi-grating $(b)$ types of surface microrelief, dipcoated with SWCNT 8 times (2) and 16 times (3).
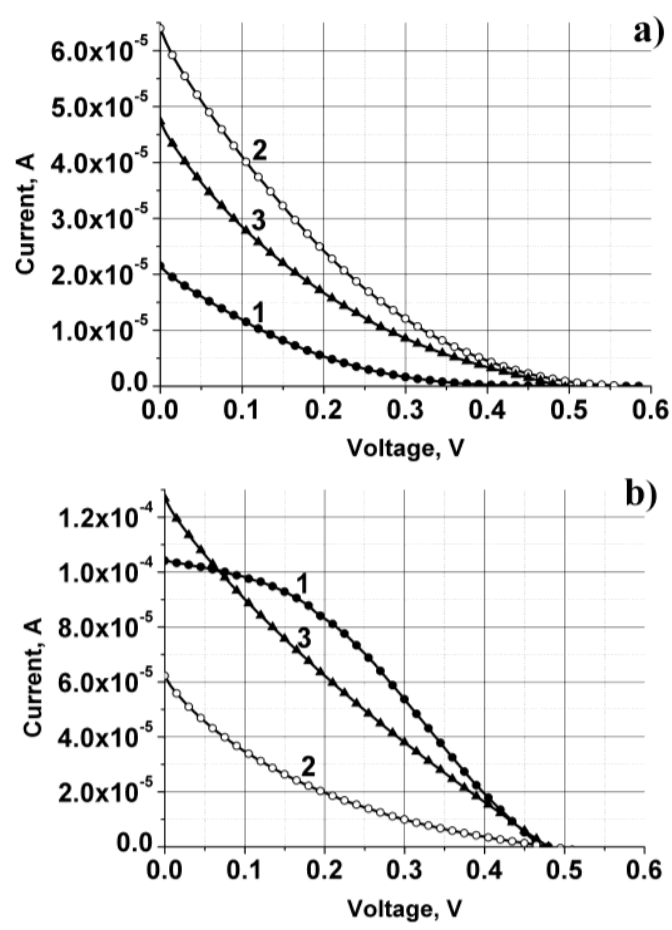

Fig. 6. The light current-voltage characteristics for the diode structures $\left(0.5 \mathrm{~mm}^{2}\right)$ with the flat $(a)$ and textured $(b)$ interfaces of quasi-grating type at AM0 simulated illumination: $\mathrm{Au} / \mathrm{GaAs}$ (1), Au/SWCNT (8 dips)/PVP/GaAs (2) and Au/SWCNT (16 dips)/PVP/GaAs (3).
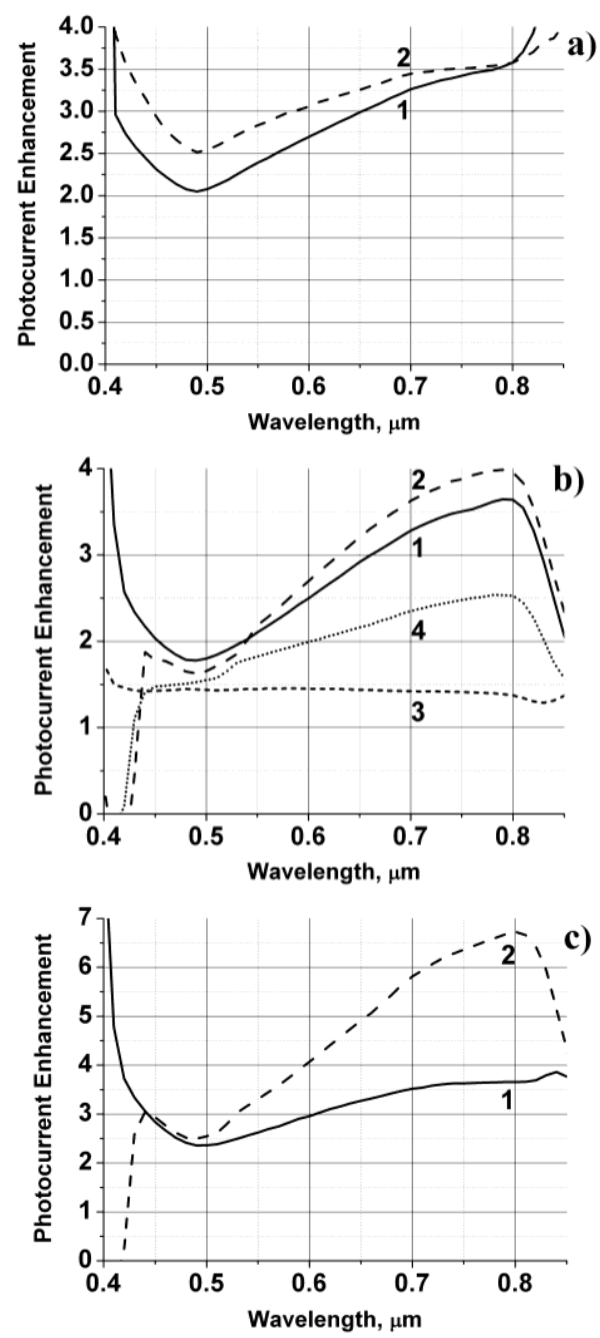

Fig. 7. a) Spectra of the short-circuit photocurrent enhancement due to microtextured interface in $\mathrm{Au} / \mathrm{GaAs}$ barrier structures: of dendrite (l) and quasi-grating (2) types. b) Spectra of the short-circuit photocurrent enhancement due to introduction of SWCNTs layer by dip coating repeated 8 times $(1,3)$ and 16 times $(2,4)$ for the structures with flat surface $(1,2)$ and surface microrelief of quasi-grating type $(3,4)$. c) Spectra of the short-circuit photocurrent enhancement due to the total effect of the surface microtexturing (of quasi-grating type) and introduction of SWCNTs by dip coating repeated 8 times (1) and 16 times (2).

The total enhancement effect of microtexturing the interface and deposition of SWCNT layer on the photocurrent of $\mathrm{Au} / \mathrm{GaAs}$ photovoltaic structures may be evaluated from the dependences shown in Fig. 7. It is seen that SWCNT layer permits to considerably increase the photocurrent enhancement caused by surface texturing at an optimal choice of its thickness.

\section{Conclusions}

The enhancement of the photocurrent, especially in the long-wave range, has been found for $\mathrm{Au} / n$-GaAs barrier structures both with flat and microtextured surfaces due 
to introduced SWCNT layers. Its mechanism has been analyzed with taking into account optical and electrical characteristics of the structures. The greatest effect of photocurrent enhancement has been observed for structures with SWCNTs deposited on flat substrates. At the same time, introduction of SWCNT/PVP layer with the optimized thickness was shown to considerably enhance the effect of the photocurrent increase caused by GaAs surface microtexturing. The effect of the additional photocurrent enhancement is mainly caused by increasing its lateral component due to enlargement of the current collection area and possible increase of the light transmittance into semiconductor, owing to scattering by nanotubes (light trapping) dependent on SWCNT nanolayer thickness and homogeneity.

\section{References}

1. 1. M.S. Dresselhaus, G. Dresselhaus, P.C. Eklund, Science of Fullerenes and Carbon Nanotubes. Academic Press, 1996.

2. M.S. Dresselhaus, G. Dresselhaus, P. Avouris, Carbon Nanotubes: Synthesis, Structure, Properties and Applications, 1st ed. Springer-Verlag, Heidelberg, Germany, 2001.

3. N.L. Dmitruk, O.Yu. Borkovskaya, I.B. Mamontova et al., Effect of $\mathrm{C}_{60}$ interfacial layer on photoelectrical characteristics of metal/Si photovoltaic structures // Proc. $22^{\text {nd }}$ European Photovoltaic Solar Energy Conference and Exhibition, Milan, Italy, September 3-7, 2007, p. 575-578.
4. J. Van de Lagemaat, T.M. Barnes, G. Cumbles et al., Organic solar cells with carbon nanotubes replacing $\mathrm{In}_{2} \mathrm{O}_{3}: \mathrm{Sn}$ as transparent electrode // Appl. Phys. Lett. 88(23), p. 233503-233505 (2006).

5. N.L. Dmitruk, O.Yu. Borkovskaya, T.S. Havrylenko et al., Effect of single-wall carbon nanotubes layer on photoelectric response of $\mathrm{Au} / \mathrm{Si}$ photovoltaic structures // Physica E, 51, p. 75-78 (2013).

6. H. Kataura, Y. Kumazawa, Y. Maniwa, I. Umezu, S. Suzuki, Y. Ohtsuka, Y. Achiba, Optical properties of single-wall carbon nanotubes // Synth. Metals, 103, p. 2555-2558 (1999).

7. M.Y. Zavodchikova, T. Kulmala, A.G. Nasibulin, V. Ermolov, S. Franssila, K. Grigoras, E.I. Kauppinen, Carbon nanotube thin film transistors based on aerosol methods // Nanotechnology 20, 085201085209 (2009).

8. L. Ren, Sh. Wang, Tailoring optical and electrical properties of carbon nanotube networks for photovoltaic applications // Carbon, 48, p. 4397 4402 (2010).

9. N.L. Dmitruk, O.Yu. Borkovskaya, I.B. Mamontova, S.V. Mamykin, Texturized interface as a basis of surface-barrier hetero-structures for solar cells application // Solar Energy Materials and Solar Cells, 60, p. 379-390 (2000).

10. S. Malynych, I. Luzinov, G. Chumanov, Poly(vinyl pyridine) as a universal surface modifier for immobilization of nanoparticles // J. Phys. Chem. $B$, 106, p. 1280-1285 (2002). 